\title{
Minimum-uncertainty coherent states of the hyperbolic and trigonometric Rosen-Morse oscillators
}

\section{Marcin Molski ${ }^{1}$ [D}

Received: 20 December 2020 / Accepted: 11 August 2021 / Published online: 21 August 2021

(c) The Author(s) 2021

\begin{abstract}
A mixed supersymmetric-algebraic approach is employed to generate the minimum uncertainty coherent states of the hyperbolic and trigonometric Rosen-Morse oscillators. The method proposed produces the superpotentials, ground state eigenfunctions and associated eigenvalues as well as the Schrödinger equation in the factorized form amenable to direct treatment in the algebraic or supersymmetric scheme. In the standard approach the superpotentials are calculated by solution of the Riccati equation for the given form of potential energy function or by differentiation of the ground state eigenfunction. The procedure applied is general and permits derivation the exact analytical solutions and coherent states for the most important model oscillators employed in molecular quantum chemistry, coherent spectroscopy (femtochemistry) and coherent nonlinear optics.
\end{abstract}

Keywords Coherent states · Rosen-Morse I and II oscillators · Algebraic methods · Supersymmetric method

\section{Introduction}

The coherent states discovered by Schrödinger in 1926 [1] are usually defined in the following manner [2]: (1) they are eigenstates of the annihilation operator, (2) they minimize the generalized position-momentum uncertainty relation and (3) they arise from the operation of a unitary displacement operator to the ground state of the oscillator. Definitions: (1) refers to Barut-Girardello [3] or, after generalization, Gazeau-Klauder states [4], (2) stands for Nieto-Simmons [5] states, whereas (3) represents Klauder-Perelomov coherent states [6-8]. From the metodological point of view, the coherent states can be constructed using irreducible representations of a Lie group [8], an algebraic approach [9], supersymmetric quantum mechanics (SUSYQM) [10]

Marcin Molski

mamolski@amu.edu.pl

1 Quantum Chemistry Department, Faculty of Chemistry, Adam Mickiewicz University of Poznań, ul. Uniwersytetu Poznańskiego 8, 61-614 Poznan, Poland 
or mixed algebraic-supersymmetric approach proposed by Molski [11]. In the latter case, the method generates not only well-known coherent states of the harmonic and Morse [12] oscillators but also so far unknown coherent states of the Wei [13], generalized Morse and modified Kratzer-Fues [14, 15] ones. In this work, we extend the research area to construct the minimum-uncertainty coherent states of the hyperbolic Rosen-Morse (HRM) [16] and trigonometric Rosen-Morse (TRM) oscillators [17]. Those models are widely used in many areas of exact sciences including quantum chromodynamics (quark interactions) [18], $\mathrm{N}$-fold supersymmetries in Schrödinger, Pauli and Dirac equations [19], SUSYQM [17, 20], the theory of molecular vibrations [16, 21] and other fields of modern chemistry [22, 23] and physics [24].

Although the Gazeau-Klauder states of the TRM oscillator have already been constructed by Chenaghlou and Faizy [25], the correctness of their procedure employed has been criticized by Fakri and Dehghani [26]. They proved that the claimed coherency for the TRM wavefunctions cannot actually exist. The minimum-uncertainty coherent states for the symmetric HRM oscillator have been obtained also by Nieto and Simmons [27] but they employed a simplified form $V(q)=V_{0} \tanh (a q)^{2}$ of the original Rosen-Morse potential $V(q)=B \tanh (q / d)-C \operatorname{sech}(q / d)^{2}$. Hence, the problem of construction of coherent states of the HRM and TRM oscillators still remains to be solved. In view of this, the main objective of the present study is construction of the coherent states of the HRM and TRM oscillators, which minimize the generalized position-momentum uncertainty relation. We shall also be concerned with obtaining exact analytical solutions and coherent states for the rotating TRM oscillator with possible application in generating coherent states of the hydrogen atom in the expanding universe or the strong interactions of quarks - the fundammental constituent of hadrons

\section{The method}

In the standard algebraic or supersymmetric treatment, the vibrational dimensionless Schrödinger equation $(\hbar=m=1)$

$$
\left[\frac{1}{2} \hat{p}^{2}+V(q)-E_{0}\right]\left|n>=\Delta E_{n 0}\right| n>, \hat{p}=-i \frac{d}{d q}
$$

is solved using a factorization procedure converting Eq. (1) to the form

$$
\begin{gathered}
\hat{A}^{\dagger} \hat{A}\left|n>=\Delta E_{n 0}\right| n>,\left[\hat{A}, \hat{A}^{\dagger}\right]=-\frac{d x(q)}{d q}, \\
\hat{A}=\frac{1}{\sqrt{2}}\left[\frac{d}{d q}-x(q)\right], \hat{A}^{\dagger}=\frac{1}{\sqrt{2}}\left[-\frac{d}{d q}-x(q)\right],
\end{gathered}
$$

in which $\Delta E_{n 0}=E_{n}-E_{0}, q=r-r_{0}$ denotes a spatial variable, $r_{0}$ is an equilibrium distance whereas $\hat{A}$ and $\hat{A}^{\dagger}$ represent annihilation and creation operators defined by $x(q)$ which satisfies the commutation relation $[x(q), \hat{p}]=i d x(q) / d q$. The explicit 
form of $x(q)$ can be determined for a given form of the potential energy function, using the Riccati equation [17]

$$
V(q)-E_{0}=\frac{1}{2}\left[x^{2}(q)+\frac{d x(q)}{d q}\right] .
$$

Vital for the approach proposed is the assumption that the second term in the Riccati equation (4) can be specified in the form

$$
\frac{d x(q)}{d q}=-f(x)
$$

in which

$$
f(x)=c_{1}\left(x+c_{0} / c_{1}\right)+c_{2}\left(x+c_{0} / c_{1}\right)^{2}+\ldots
$$

is expanded into a power series of $|x(q)|<1$. Now, successive applications of the zero-, first-, second- and higher-order terms allow us to calculate $x(q)$ and then the ground state eigenfunction $\mid 0>$ by integration of Eq. (5) and annihilation equation $\hat{A} \mid 0>=0$ yielding

$$
\int \frac{d x}{f(x)}=-q, \mid 0>=\exp \left[\int x(q) d q\right]
$$

Additionally, from (4) one gets the potential $V(q)$ and the ground state eigenvalue $E_{0}$, whereas $\mid 0>$ permits the construction of the coherent states $\mid \alpha>$ being the eigenstates of the annihilation operator $\hat{A}$

$$
\hat{A}|\alpha>=\alpha| \alpha>=\alpha \mid 0>\exp (\sqrt{2} \alpha q) .
$$

It has been proved [11] that such states minimize the generalized position-momentum uncertainty relation [9]

$$
\begin{gathered}
(\Delta x(q))^{2}(\Delta p)^{2} \geq \frac{1}{4}<\alpha\left|\frac{d x(q)}{d q}\right| \alpha>^{2}, \\
\frac{d x(q)}{d q}=-i[x(q), \hat{p}]=-\left[\hat{A}, \hat{A}^{\dagger}\right],
\end{gathered}
$$

which can be specified in an alternative form

$$
(\Delta x(q))^{2}(\Delta p)^{2}=\frac{1}{4}<\alpha|f[x(q)]| \alpha>^{2}
$$

including $f(x)$ as an genuine component of the uncertainty relation (9). Hence, they satisfy two fundamental requirements established for the minimum-uncertainty coherent states of harmonic and anharmonic oscillators.

In algebraic methods, $x(q)$ is treated as a vibrational (harmonic or anharmonic) variable [9], whereas in SUSYQM $x(q)$ (with negative sign) is interpreted as a superpotential $W(q)=-x(q)$ [17], which permits conversion of the Schrödinger equation (1) to 
the factorized form (2) straightforward to analytical treatment. Hence, having obtained the ground state solution, one may derive the eigenfunctions and associated eigenvalues for excited states using supersymmetric (SUSYQM) formalism [17]. In this approach, two operators $\hat{H}$ and $\hat{H}^{\dagger}$

$$
\begin{aligned}
& \hat{H}=\frac{1}{\sqrt{2}}\left[-\frac{d}{d q}+W(q)\right] \frac{1}{\sqrt{2}}\left[\frac{d}{d q}+W(q)\right]=\hat{A}^{\dagger} \hat{A}, \\
& \hat{H}^{\dagger}=\frac{1}{\sqrt{2}}\left[\frac{d}{d q}+W(q)\right] \frac{1}{\sqrt{2}}\left[-\frac{d}{d q}+W(q)\right]=\hat{A} \hat{A}^{\dagger}
\end{aligned}
$$

form the two-component Hamiltonian [17]

$$
\hat{H}_{S}=\left[\begin{array}{cc}
\hat{H} & 0 \\
0 & \hat{H}^{\dagger}
\end{array}\right]
$$

including fermionic $\hat{H}$ and bosonic $\hat{H}^{\dagger}$ components, respectively. They are supersymmetric partners of each other and correspond to an isospectral pair of potentials $V(q)$ and $V(q)^{\dagger}$ defined in the following manner

$$
\begin{aligned}
V(q)-E_{0} & =\frac{1}{2}\left[W(q)^{2}-\frac{d W(q)}{d q}\right], V(q)^{\dagger}-E_{0}^{\dagger}=\frac{1}{2}\left[W(q)^{2}+\frac{d W(q)}{d q}\right], \\
V(q)^{\dagger} & =V(q)+\frac{d W(q)}{d q}, \text { for } E_{0}=E_{0}^{\dagger} .
\end{aligned}
$$

The Hamiltonians $\hat{H}$ and $\hat{H}^{\dagger}$ as well as their eigenfunctions $\psi_{n}=\left|n>, \psi_{n}^{\dagger}=<n\right|$ and the associated eigenvalues $E_{n}, E_{n}^{\dagger}$ are interrelated via the well-known manner $(n>0)$ [17]

$$
\begin{array}{r}
\hat{H}\left(\hat{A}^{\dagger} \psi_{n}^{\dagger}\right)=E_{n}^{\dagger}\left(\hat{A}^{\dagger} \psi_{n}^{\dagger}\right) \hat{H}^{\dagger}\left(\hat{A} \psi_{n}\right)=E_{n}\left(\hat{A} \psi_{n}\right) \\
E_{n}^{\dagger}=E_{n+1}, \psi_{n}^{\dagger}=\left(E_{n+1}\right)^{-1 / 2} \hat{A} \psi_{n+1}, \psi_{n+1}=\left(E_{n}^{\dagger}\right)^{-1 / 2} \hat{A}^{\dagger} \psi_{n}^{\dagger} .
\end{array}
$$

Hence, knowing all eigenfunctions of $\hat{H}$ one can determine the eigenfunctions of $\hat{H}^{\dagger}$ by making use of the operator $\hat{A}$, and vice versa application of $\hat{A}^{\dagger}$ makes it possible to reconstruct all eigenfunctions of $\hat{H}$ from those of $\hat{H}^{\dagger}$ except for the ground state $E_{0}$. In view of this, the combination of algebraic and SUSYQM methodology permits obtaining a full quantum characteristic of the oscillators including ground and excited states. To this aim we work in the following scheme

$$
f(x) \longrightarrow x(q), W(q) \longrightarrow V(q), V(q)^{\dagger}, E_{0},|0>\longrightarrow| n>,<n \mid, E_{n}, E_{n}^{\dagger} \text { for } n>0 .
$$

In the case of the shape-invariant potentials, the unnormalized excited states $|n\rangle$ and associated eigenvalues $E_{n}$ can be generated by multiple action of the creation operator on the ground state $\mid 0>$ [17]

$$
\left|n>=\sqrt{2^{n}}\left(\hat{A}^{\dagger}\right)^{n}\right| 0>
$$


and substitution of $\mid n>$ into the wave equation (1), which yields the energy spectrum $E_{n}$.

In the algebraic approach, the eigenfunctions of the excited states $\mid n>$ are calculated in the form of a product of the ground state solution and the polynomial function $F(u)_{n}$

$$
\left|n>=F(u)_{n}\right| 0>
$$

expressed in an adequate variable $u(q)$ [17]. $F(u)_{n}$ usually represents the generalized hypergeometric function including Bessel, Jacobi (Legendre, Gegenbauer, Chebychev), Laguerre or Romanovski functions as the special cases. Introducing a trial function (17) into (1) one gets the general equation [28, 29]

$$
\left(a q^{2}+b q+c\right) F(u)_{n}^{\prime \prime}+(d q+e) F(u)_{n}^{\prime}-\lambda_{n} F(u)_{n}=0, n=0,1,2 \ldots
$$

generating a polynomial term in the form depending on $a, b, c, d, e$ parameters. Application of the proper boundary conditions to solutions of (18) permits calculation of the eigenvalues $E_{n}$ of (1) in the quantized form.

\section{The HRM oscillator}

A detailed mathematical analysis of the impact of $f(x)$ on the form of quantum solutions generated in the mixed scheme reveals [11] that they depend not only on different powers $n=0,1,2$ of the truncated series (6) but also on the relations between parameters $c_{0}, c_{1}, c_{2}$ and the presence or absence of the term $\left(x+c_{0} / c_{1}\right)^{l}$ for $l<n$ in the series (6). In this work, we, introduce another modification of the expansion (6), assuming its form as

$$
f(x)=c_{1}\left(x \pm c_{0} / c_{1}\right) \pm c_{2}\left(x \pm c_{0} / c_{1}\right)^{2}+\ldots c_{i}>0
$$

Inserting a special case of (19)

$$
f(x)=c_{0}-c_{2}\left(x-c_{0} / c_{1}\right)^{2}
$$

into the first of the Eqs.(7) and then integrating it with respect to $x$-coordinate yields

$$
-\left(c_{0} c_{2}\right)^{-1 / 2} \tanh ^{-1}\left[\frac{c_{2}\left(c_{0}-c_{1} x\right)}{c_{1} \sqrt{c_{0} c_{2}}}\right]=-q .
$$

The equation derived above can be reverted generating $x(q)$ in the $q$-dependent form

$$
x(q)=-\sqrt{\frac{c_{0}}{c_{2}}} \tanh \left(q \sqrt{c_{0} c_{2}}\right)+\frac{c_{0}}{c_{1}} .
$$

Having introduced $x(q)$ one may pass to calculate $\mid 0>, V(q)$ and $E_{0}$ by taking advantage of Eqs. (7) and (4). In the first case one gets 


$$
\mid 0>=\exp \left(\frac{c_{0}}{c_{1}} q\right)\left[\tanh \left(q \sqrt{c_{0} c_{2}}\right)^{2}-1\right]^{\frac{1}{2 c_{2}}}
$$

or equivalently, after trigonometric simplification employing identity $1-\tanh ^{2}(z)=\cosh ^{-2}(z)=\operatorname{sech}(z)^{2}$

$$
\mid 0>=N_{0} \exp \left(\frac{c_{0}}{c_{1}} q\right) \cosh \left(q \sqrt{c_{0} c_{2}}\right)^{-\frac{1}{c_{2}}} .
$$

Here $N_{0}=i^{1 / c_{2}}$ in which $i=\sqrt{-1}$ is an imaginary number. Taking advantage of the Eqs. (4) and (22) as well as the first derivative of $x(q)$

$$
\frac{d x(q)}{d q}=-c_{0}\left[1-\tanh \left(q \sqrt{c_{0} c_{2}}\right)^{2}\right]=-c_{0} \operatorname{sech}\left(q \sqrt{c_{0} c_{2}}\right)^{2}
$$

one may convert the Schrödinger equation (1) to the form

$$
\left[-\frac{1}{2} \frac{d^{2}}{d q^{2}}-\frac{c_{0}}{c_{1}} \sqrt{\frac{c_{0}}{c_{2}}} \tanh \left(q \sqrt{c_{0} c_{2}}\right)-\frac{1}{2}\left(\frac{c_{0}}{c_{2}}+c_{0}\right) \operatorname{sech}\left(q \sqrt{c_{0} c_{2}}\right)^{2}+\frac{1}{2}\left(\frac{c_{0}^{2}}{c_{1}^{2}}+\frac{c_{0}}{c_{2}}\right)\right] \mid 0>=0 .
$$

Redefining constants appearing in Eqs. (24)-(26)

$$
c_{0}=\frac{b_{0}}{d^{2}}, c_{1}=\frac{b_{0}}{a_{0} d}, c_{2}=\frac{1}{b_{0}}, \sqrt{c_{0} c_{2}}=\frac{1}{d},
$$

one may convert (24) and (26) to the forms oryginally obtained by Rosen and Morse [16]

$$
\begin{gathered}
\mid 0>=N_{0} \exp \left(a_{0} z\right) \cosh (z)^{-b_{0}} \\
\left\{-\frac{1}{2} \frac{d^{2}}{d q^{2}}+B \tanh (q / d)-C \operatorname{sech}(q / d)^{2}-E_{0}\right\} \mid 0>=0,
\end{gathered}
$$

in which

$$
\begin{aligned}
E_{0} & =\frac{1}{2}\left(\frac{c_{0}^{2}}{c_{1}^{2}}+\frac{c_{0}}{c_{2}}\right)=\left(a_{0}^{2}+b_{0}^{2}\right) / 2 d^{2}, \\
& -\frac{c_{0}}{c_{1}} \sqrt{\frac{c_{0}}{c_{2}}}=-\frac{a_{0} b_{0}}{d^{2}}=B, \frac{1}{2}\left(\frac{c_{0}}{c_{2}}+c_{0}\right)=\frac{b_{0}^{2}+b_{0}}{2 d^{2}}=C .
\end{aligned}
$$

The eigenfunction $\mid 0>$ and eigenvalue $E_{0}$ derived represent the exact analytical ground state solutions for the HRM potential. In particular, the energy formula (30) is a special case of the more general expression

$$
E_{n}=-\frac{\hbar^{2}}{2 m d^{2}}\left(a_{n}^{2}+b_{n}^{2}\right), b_{n}=\sqrt{\gamma+1 / 4}-n-1 / 2, a_{n}=-\beta /\left(2 b_{n}\right) n=0,1,2 \ldots
$$


obtained together with excited states $\mid n>$

$$
\begin{gathered}
\mid n>=N_{n} \exp \left(a_{n} q / d\right) \cosh (q / d)^{-b_{n}} F_{n}\left(-n,(4 \gamma+1)^{1 / 2}-n ; a_{n}+b_{n}+1 ; u\right) \\
\left.u=\frac{1}{2}[1+\tanh (q / d])\right]
\end{gathered}
$$

in the algebraic scheme by Rosen and Morse [16]. Here, $N_{n}$ is normalizattion constant reported in [30], $F_{n}(u)$ stands for the hypergeometric function expressed in the coordinate $u(q)$ whereas

$$
\beta=\frac{2 m d^{2} B}{\hbar^{2}}=-2 a_{0} b_{0}, \gamma=\frac{2 m d^{2} C}{\hbar^{2}}=b_{0}\left(b_{0}+1\right)
$$

Having derived $\mid 0>$ with $x(q)$ appearing in the annihilation operator $\hat{A}$ one may construct the coherent states of the HRM oscillator employing the formula (8)

$$
|\alpha\rangle=\exp \left(a_{0} q / d\right) \cosh (q / d)^{-b_{0}} \exp (\sqrt{2} \alpha q)
$$

which are eigenstates of the annihilation operator

$$
\frac{1}{\sqrt{2}}\left[\frac{d}{d q}+\frac{b_{0}}{d} \tanh (q / d)-\frac{a_{0}}{d}\right]|\alpha\rangle=\alpha|\alpha\rangle,
$$

and minimize the generalized uncertainty relation (10)

$$
[\Delta x(q)]^{2}(\Delta p)^{2}=\frac{1}{4}\langle\alpha|f(x)| \alpha\rangle^{2}, f(x)=b_{0} / d^{2}-\left(x-a_{0} / d\right)^{2} / b_{0}
$$

including $x(q)$ defined by (22).

To obtain the full SUSYQM characteristics of the HRM oscillator we need the superpotential $W(q)$ and the partner potential $V(q)^{\dagger}$ defined by (13). Taking advantage of Eqs. (22), (25) and (27) one qets

$$
\begin{gathered}
W(q)=-x(q)=\frac{b_{0}}{d} \tanh \left(\frac{q}{d}\right)-\frac{a_{0}}{d} \frac{d W(q)}{d q}=\frac{b_{0}}{d^{2}} \operatorname{sech}\left(\frac{q}{d}\right)^{2} \\
V(q)=-\frac{a_{0} b_{0}}{d^{2}} \tanh \left(\frac{q}{d}\right)-\frac{b_{0}\left(b_{0}+1\right)}{2 d^{2}} \operatorname{sech}\left(\frac{q}{d}\right)^{2}=B \tanh \left(\frac{q}{d}\right)-C \operatorname{sech}\left(\frac{q}{d}\right)^{2} \\
V(q)^{\dagger}=-\frac{a_{0} b_{0}}{d^{2}} \tanh \left(\frac{q}{d}\right)-\frac{b_{0}\left(b_{0}-1\right)}{2 d^{2}} \operatorname{sech}\left(\frac{q}{d}\right)^{2}=B \tanh \left(\frac{q}{d}\right)-\left(C-b_{0} d^{-2}\right) \operatorname{sech}\left(\frac{q}{d}\right)^{2}
\end{gathered}
$$

indicating that the potentials $V(q)^{\dagger}$ and $V(q)$ are interrelated via substitution $C \longrightarrow C-b_{0} d^{-2}$ in $V(q)$, hence the HRM potential belongs to the class of shape invariant functions [17]. 


\section{The TRM oscillator}

The methodology proposed can also be applied to obtain quantum solutions for the TRM oscillator [17] by taking advantage of the generating function (19) in the form

$$
f(x)=a+a^{-1}\left[x(q)+b a^{-1}\right]^{2}
$$

defined directly by the potential parameters instead of $c_{i}$ included in (19). To simplify mathematical calculations, we can also use the Maple processor for symbolic calculations which enables quick solution of the Eq. (5) given in the form

$$
\frac{d x(q)}{d q}+a+a^{-1}\left[x(q)+b a^{-1}\right]^{2}=0
$$

yielding

$$
x(q)=a \cot \left(q+C^{\prime}\right)-b a^{-1} .
$$

Here, $C^{\prime}$ stands for a constant to be calculated in the next step of derivation of the ground state eigenfunction of the TRM oscillator by making use of

$$
\begin{aligned}
& \left.|0\rangle=\exp \left[\int_{q} x(q) d q+C\right]=N_{0} \exp \left\{a \ln \left[\sin \left(q+C^{\prime}\right)\right]-b a^{-1} q\right)\right\}= \\
& N_{0} \exp \left[-b q a^{-1}\right] \sin \left(q+C^{\prime}\right)^{a},
\end{aligned}
$$

in which $N_{0}=\exp [C]$ is normalizattion constant whose explicit form is reported in [31], whereas the potential and ground state eigenvalue are determinable using (43)

$$
\begin{aligned}
& V(q)-E_{0}=\frac{1}{2}\left[x(q)^{2}+\frac{d x(q)}{d q}\right]=\frac{1}{2}\left\{\left[a \cot \left(q+C^{\prime}\right)-b a^{-1}\right]^{2}-a \sin \left(q+C^{\prime}\right)^{-2}\right\} \\
= & \frac{a(a-1)}{2} \csc \left(q+C^{\prime}\right)^{2}-b \cot \left(q+C^{\prime}\right)-\frac{1}{2}\left(a^{2}-\frac{b^{2}}{a^{2}}\right) .
\end{aligned}
$$

In the equations specified above, $q$-variable is defined in the range $0 \leq q \leq \pi$ hence we can calculate the constant $C^{\prime}$ by making use of the boundary condition $\mid 0>=0$ for $q=0$ providing $C^{\prime}=0$. Having derived the ground state eigenfunction (44) one may generate the excited states and associated eigenvalues

$$
\begin{gathered}
|n\rangle=\exp \left(-b q(a+n)^{-1}\right) \sin (q)^{a+n} F(u)_{n}, u=\cot (q) \\
E_{n}=\frac{1}{2}\left[(a+n)^{2}-\frac{b^{2}}{(a+n)^{2}}\right] n=0,1,2 \ldots
\end{gathered}
$$

using algebraic approach or SUSYQM nethod described in details in [20]. The polynomial function $F_{n}(u)$ in Eq. (46) is the solution to the equation 


$$
\left(1+u^{2}\right) F_{n}^{\prime \prime}(u)+\left(\alpha_{n}+2 \beta_{n} u\right) F_{n}^{\prime}(u)+\left[\beta_{n}\left(\beta_{n}-1\right)-a(a-1)\right] F_{n}(u)=0
$$

in which

$$
\beta_{n}=-(a+n), \alpha_{n}=\frac{2 b}{a+n}, n=0,1,2 \ldots
$$

and explicit forms of $F_{n}(u)$ are presented in [20].

Proceeding in a similar manner as in the case of the HRM oscillator, we can construct the minimum uncertainty coherent states taking advantage of the ground state solution (44) in an arbitrary normalization

$$
|\alpha\rangle=\exp \left(-b q a^{-1}\right) \sin (q)^{a} \exp (\sqrt{2} \alpha q),
$$

which are eigenstates of the annihilation operator

$$
\frac{1}{\sqrt{2}}\left[\frac{d}{d q}-a \cot (q)+b a^{-1}\right]|\alpha\rangle=\alpha|\alpha\rangle
$$

and minimize the generalized uncertainty relation (9)

$$
[\Delta x(q)]^{2}(\Delta p)^{2}=\frac{1}{4}\langle\alpha|f(x)| \alpha\rangle^{2}, f(x)=a+a^{-1}\left[x(q)+b a^{-1}\right]^{2}
$$

including $x(q)$ specified by (43).

The methodology applied to TRM oscillator can be extended to include rotational degree of freedom by making use of the generating function (41) with

$$
a=l+1, l=0,1,2 \ldots
$$

providing ground state eigenfunction

$$
\mid 0 l>=N_{0} \exp \left[-b(l+1)^{-1} q\right] \sin \left(q+C^{\prime}\right)^{l+1}, N_{0}=\exp [C],
$$

potential and $l$-dependent vibrational ground state eigenvalue

$$
\begin{aligned}
& V(q)-E_{0}=\frac{1}{2}\left\{\left[(l+1) \cot \left(q+C^{\prime}\right)-b(l+1)^{-1}\right]^{2}-(l+1) \sin \left(q+C^{\prime}\right)^{-2}\right\}= \\
& \frac{l(l+1)}{2} \csc \left(q+C^{\prime}\right)^{2}-b \cot \left(q+C^{\prime}\right)-\frac{1}{2}\left[(l+1)^{2}-b^{2}(l+1)^{-2}\right]
\end{aligned}
$$

in which $C^{\prime}=0$ as $\mid 0 l>=0$ for $q=0$. The potential energy function $V(q)$ specified in a slightly changed form

$$
2 V(q)=l(l+1) \csc (q)^{2}-2 b \cot (q) l=0,1,2 \ldots q=r / d .
$$

has been employed to formulate [32] the hydrogen atom problem in Einstein's closed universe $R^{1} \otimes S^{3}$ with a positive constant curvature, to describe [33] quantum motion of a charge dipole perturbed by another charge dipole interacting via modified Coulomb's potential, to model [18] the strong interactions of 
quarks - fundammental constituents of hadrons and other areas of modern chemistry [22, 23] and physics [24].

Having derived the vibrational ground state eigenfunction one may calculate the exicited eigenstates using the algebraic approach providing [18]

$$
\begin{aligned}
|k l\rangle=N_{k, l} \sin (q)^{k+1} & \exp \left(-\frac{b q}{k+1}\right) F_{n}^{2 b /(k+1),-(k+1)}[\cot (q)], \\
k & =0,1,2 . ., l=0,1,2 \ldots, k, n=k-l \\
E_{k} & =(k+1)^{2}-\frac{b^{2}}{(k+1)^{2}}
\end{aligned}
$$

in which $F_{k-l}^{2 b /(k+1),-(k+1)}[\cot (q)]$ stands for the Romanovski polynomials. For the vibrational ground state $n=k-l=0$ and rotational $l$-state one gets

$$
|l l\rangle=N_{l, l} \sin (q)^{l+1} \exp \left(-\frac{b q}{l+1}\right)
$$

hence, the minimum uncertainty coherent states for the rotatating TRM oscillator

$$
|\alpha l\rangle=\sin (q)^{l+1} \exp \left(-b(l+1)^{-1} q\right) \exp (\sqrt{2} \alpha q)
$$

are the eigenstates of the $l$-dependent annihilation operator

$$
\frac{1}{\sqrt{2}}\left[\frac{d}{d q}-(l+1) \cot (q)+b(l+1)^{-1}\right]|\alpha l\rangle=\alpha|\alpha l\rangle,
$$

and minimize the generalized uncertainty relation (9) adopted to the rotational case

$$
[\Delta x(q)]^{2}(\Delta p)^{2}=\frac{1}{4}\langle\alpha l|f(x)| \alpha l\rangle^{2}, f(x)=(l+1)+(l+1)^{-1}\left[x(q)+b(l+1)^{-1}\right]^{2} .
$$

Here, $x(q)$ defined by (43) with $a=l+1$, denotes the trigonometric Rosen-Morse variable. The formulae specified above are consistent with those obtained for the rotational coherent states of the Kratzer-Fues oscillator [34]. Hence, one may expect that the quark-diquark systems can be excited via resonant interactions with the laser beam producing a strong electromagnetic pulse [35]. It can generate in molecules and nuclei [36-39] peculiar coherent effects such as: self-induced transparency, excitation of coherent superposition of rotational states, periodic alternations of the refractive index, soliton formation and others.

The pseudopotential $W(q)$ and the partner potential $V(q)^{\dagger}$ vital for the SUSYQM approach can be calculated by taking advantage of Eqs. (5) and (43) $(a=l+1)$ yielding

$$
W(q)=-x(q)=-(l+1) \cot (q)+b(l+1)^{-1} \frac{d W(q)}{d q}=\frac{(l+1)}{\sin (q)^{-2}}
$$




$$
V(q)=\frac{l(l+1)}{2} \csc (q)-b \cot (q) V(q)^{\dagger}=\frac{(l+1)(l+2)}{2} \csc (q)-b \cot (q)
$$

The equation presented above reveals that the partner potential $V(q)^{\dagger}$ is interrelated with $V(q)$ via substitution $l \longrightarrow l+1$ in $V(q)$, hence the TRM potential for rotating systems belongs to the class of shape invariant functions [17].

\section{Conclusions}

Coherent states of anharmonic oscillators have been constructed using several alternative approaches applied to Morse [5, 9, 40-42], Pöschl-Teller [43], hydrogen atom [44, 45], double-well and linear(gravitational) [27] as well as unusual potentials [46] taken as examples. In the present study, the minimum-uncertainty coherent states of the HRM and TRM oscillators have been obtained within the mixed supersymmetric-algebraic method, which permits generating not only the coherent states of the well-known anharmonic oscillators but also deriving superpotentials without necessity of using the ground-state wave function or solving the Riccati equation for a given form of the potential energy function. Consequently, the Schrödinger equation can be derived in the factorized form amenable to direct treatment in the algebraic or SUSYQM scheme [47]. In the standard approach, the superpotentials are generated by solution of the Riccati equation (4) for the selected potentials $V(q)$ [17], hence, this procedure is difficult to follow. The new approach permits derivation of the potentials and associated superpotentials starting from Eq. (5) in which the term $f(x)$ is expanded into a power series of $x(q)$ and then used to generate the coherent states for different orders and forms of the expansion (6). In this way one may construct exact analytical solutions and associated coherent states for the most important model oscillators employed in molecular quantum mechanics, coherent spectroscopy (femtochemistry) and coherent nonlinear optics embracing a wide class of problems under consideration [48-51]. From the metodological point of view, the method presented can be considered as a generalization [52,53] of the phenomenological university (PU) theory $[54,55]$ to include the quantum systems. Hence, the results obtained in this work permit enriching the quantum class $Q_{2}$ (second-order expansion of $f(x)$ ) of PU by the new subclasses $Q_{2}^{3}$ and $Q_{2}^{4}$. In view of this the final classification of the second order PU embraces the following subclasses: $Q_{2}^{0}$ for Wei [13], $Q_{2}^{1}$ for Hulthen [56, 57], $Q_{2}^{2}$ for Kratzer-Fues [14, 15], $Q_{2}^{3}$ for HRM [16] and $Q_{2}^{4}$ for TRM [17] oscillators, respectively.

\section{Compliance with ethical standards}

Conflict of interest The authors declare that they have no conflict of interest.

Open Access This article is licensed under a Creative Commons Attribution 4.0 International License, which permits use, sharing, adaptation, distribution and reproduction in any medium or format, as long as you give appropriate credit to the original author(s) and the source, provide a link to the Creative 
Commons licence, and indicate if changes were made. The images or other third party material in this article are included in the article's Creative Commons licence, unless indicated otherwise in a credit line to the material. If material is not included in the article's Creative Commons licence and your intended use is not permitted by statutory regulation or exceeds the permitted use, you will need to obtain permission directly from the copyright holder. To view a copy of this licence, visit http://creativecommons.org/ licenses/by/4.0/.

\section{References}

1. E. Schrödinger, Naturwiss. 14, 664 (1926)

2. W.-M. Zhang, D.H. Feng, R. Gilmore, Rev. Mod. Phys. 62, 867 (1990)

3. A.O. Barut, L. Girardello, Commun. Math. Phys. 21, 41 (1971)

4. J.P. Gazeau, J.R. Klauder, J. Phys. A 32, 123 (1999)

5. M.M. Nieto, L.M. Simmons, Phys. Rev. Lett. 41, 207 (1978)

6. J.R. Klauder, J. Math. Phys. 4, 1055 (1963)

7. J.R. Klauder, J. Math. Phys. 4, 1058 (1963)

8. A.M. Perelomov, Commun. Math. Phys. 26, 222 (1972)

9. I.L. Cooper, J. Phys. A Math. Gen. 25, 1671 (1992)

10. M.G. Benedict, B. Molnar, Phys. Rev. A 60, R1737 (1999)

11. M. Molski, J. Phys. A Math. Gen. 42, 165301 (2009)

12. P.M. Morse, Phys. Rev. 34, 57 (1929)

13. H. Wei, Phys. Rev. A 42, 2524 (1990)

14. A. Kratzer, Z. Phys. 3, 289 (1920)

15. E. Fues, Ann. Phys. Paris 80, 281 (1926)

16. N. Rosen, P.M. Morse, Phys. Rev. 42, 210 (1932)

17. F. Cooper, A. Khare, U. Sukhatme, Phys. Rep. 2(51), 267 (1995)

18. C.B. Compean, Eur. Phys. J. A 33, 1 (2007)

19. C.-L. Ho, T. Tanaka, Ann. Phys. 321, 1375 (2006)

20. C.B. Compean, M. Kirchbach, J. Phys. A: Math. Gen. 39, 547 (2006)

21. G.-D. Zhang, J.-Y. Liu, L.-H. Zhang, W. Zhou, C.-S. Jia, Phys. Rev. A 86, 062510 (2012)

22. H. Yanar, O. Aydoğdu, M. Salti, Mol. Phys. 114, 21 (2016)

23. M.E. Udoh, U.S. Okorie, M.I. Ngwueke, E.E. Ituen, A.N. Ikot, J. Mol. Model. 25, 170 (2019)

24. L.D. Landau, E.M. Lifschitz, The Classical Theory of Fields. Vol. 2 of Course of Theoretical Physics, 3rd edn. (Pergamon Press, Oxford, 1971)

25. A. Chenaghlou, O. Faizy, J. Math. Phys. 49, 022104 (2008)

26. H. Fakhri, A. Dehghani, J. Math. Phys. 49, 052101 (2008)

27. M.M. Nieto, L.M. Simmons, Phys. Rev. D 20, 1342 (1979)

28. M. Majed-Jamei, Integ. Transf. Spec. Funct. 13, 169 (2002)

29. W. Koepf, M. Masjed-Jamei, Integ. Transf. Spec. Funct. 17, 559 (2006)

30. G. Levai, E. Magyari, J. Phys. A: Math. Theor. 42, 195302 (2009)

31. G. Levai, Phys. Lett. A 372, 6484 (2008)

32. E. Schrödinger, Proc. Roy. Irish Acad. A. 47(1941)

33. P. Pouria, Am. J. Phys. 78, 403 (2010)

34. M. Molski, Phys. Rev. A 76(1-6), 022107 (2007)

35. L. Fonda, N. Mankoč-Borštnik, M. Rosina, Phys. Rep. 158, 159 (1988)

36. N. Mankoc-Borstnik, M. Rosina, L. Fonda, Nuovo Cimento A 53, 440 (1979)

37. L. Fonda, N. Mankoc-Borstnik, M. Rosina, Nuovo Cimento A 56, 229 (1980)

38. L. Fonda, N. Mankoc-Borstnik, Nuovo Cimento A 63, 483 (1981)

39. L. Fonda, N. Mankoc-Borstnik, M. Rosina, Nuovo Cimento D 7, 437 (1986)

40. S. Kais, R.D. Levine, Phys. Rev. A 41, 2301 (1990)

41. G.C. Gerry, Phys. Rev. A 31, 2721 (1985)

42. M. Molski, Eur. Phys. J. D 40, 411 (2006)

43. M.M. Nieto, L.M. Simmons, Phys. Rev. D 20, 1332 (1979)

44. C.C. Gerry, J. Kiefer, Phys. Rev. A 37, 665 (1988)

45. J.R. Klauder, J. Phys. A Math. Gen. 29, L293 (1996) 
46. M.M. Nieto, Mod. Phys. Lett. A 16, 2305 (2001)

47. F. Schwabl, Quantum Mechanics (Springer, Berlin, 1992)

48. G.L. Lamb, Rev. Mod. Phys. 43, 99 (1971)

49. N. Manko-Bortink, L. Fonda, B. Bortnik, Phys. Rev. A 35, 4132 (1987)

50. J.P. Heritage, T.K. Gustafson, C.H. Lin, Phys. Rev. Lett. 34, 1299 (1975)

51. N.M. Avram, Gh.E. Draganescu, C.N. Avram, J. Opt. B Quantum Semiclass Opt. 2, 214 (2000)

52. M. Molski, Phys. Lett. A. 381, 2629 (2017)

53. M. Molski, Phys. Lett. A. 382, 79 (2018)

54. P. Castorina, P.P. Delsanto, C. Guiot, Phys. Rev. Lett. 96, 188701 (2006)

55. P. Castorina, P.P. Delsanto, C. Guiot, Phys. Rev. Lett. 98, 209901 (2007)

56. L. Hulthen, Ark. Mat. Astron. Fys. A. 28, 5 (1942)

57. L. Hulthen, Ark. Mat. Astron. Fys. B. 29, 1 (1942)

Publisher's Note Springer Nature remains neutral with regard to jurisdictional claims in published maps and institutional affiliations. 